\title{
A DC bias approach to the characterisation of non-linear material parameters of piezoelectric ceramics
}

\author{
Dmitrij Dreiling $^{1}$, Nadine Feldmann ${ }^{1}$, Bernd Henning ${ }^{1}$ \\ ${ }^{1}$ Measurement Engineering Group, Paderborn University, Warburger Straße 100, 33098 Paderborn, Germany
}

\begin{abstract}
Piezoelectric materials are used for various sensor and actuator purposes. Especially in power acoustic applications piezoceramics are loaded with electrical DC voltage or mechanical pre-load, in addition to the excitation signal. Due to the lack of knowledge of material parameters for piezoceramics, particularly in this operating mode, the design of a power transducer is demanding and often strongly empirically driven. A simulation-supported design process requires that a non-linear model for the piezoelectric material behaviour is known. A first step towards this material model is the description of linear approximated material properties in different operating points. To shift this operating point a DC bias voltage is applied to various piezoelectric discs and the frequency dependent impedance is determined. The results indicate a shift of all characteristic resonance frequencies as well as changes of piezoelectric parameters directly dependent on the applied bias voltages.
\end{abstract}

Keywords: piezoelectric materials, piezoelectric properties, DC bias field, non-linear material parameters

\section{Introduction}

Recently, simulation-driven procedures for the determination of material parameters of piezoelectric materials in the linear domain have been published $[1,2]$. Nevertheless, there are methods to estimate the linear material properties for simulation-driven design processes. However those provide just rough estimate and deviate strongly regarding power acoustic applications or under external influences like a mechanical/electrical pre-load. Under these circumstances, non-linearities play a non-negligible role. There are different approaches to characterise and model those non-linearities, for example by taking non-linear second order material parameters into account $[3,4]$ or by extending the Mason model [5] with non-linear voltage sources [6]. An accurate non-linear model of piezoelectricity and a better understanding could be used to increase the efficiency of ultrasonic transducers or to avoid undesired effects such as depolarisation.

A common way to determine electromechanical properties is to linearise in an operating point, to estimate the values by measuring the impedance of a specimen and to detect characteristic frequencies [7]. A DC bias field applied to a piezoceramic shifts the operating point and influences the material parameters $[3,4,8]$. Li et al. investigate the relation between the influence of external bias field or temperature on material parameters and draw the conclusion that this effect can be advantageously used for the tuning of piezoelectric properties [9]. By one-dimensional considerations for a plate resonator with finite thickness, $[3,4]$ provide a possibility to determine material parameters from a resonance frequency shift. In addition, they derive equations for the determination of nonlinear parameters by changing the operating point. This paper follows the same approach but for piezoelectric discs with adapted assumptions and modified equations. DC bias fields of various strengths are used to shift the operating point of the samples and estimate different linear material parameters (figure 1). Finally, these parameters can be used to estimate the non-linear material parameters.

\section{Mathematical description}

\subsection{Linear approximation for material properties}

Commonly, in the small signal range the relationship between the mechanical quantities, mechanical stress $\boldsymbol{T}$ and strain $\boldsymbol{S}$, and the electrical quantities, electric field $\boldsymbol{E}$ and dielectric displacement $\boldsymbol{D}$, can be regarded as approximately linear. Therefore the following constitutive equations (in Voigt notation) can be assumed

$$
\begin{aligned}
S & =\boldsymbol{s}^{E} \boldsymbol{T}+d^{\mathrm{t}} \boldsymbol{E}, \\
D & =d \boldsymbol{T}+\varepsilon^{T} E,
\end{aligned}
$$




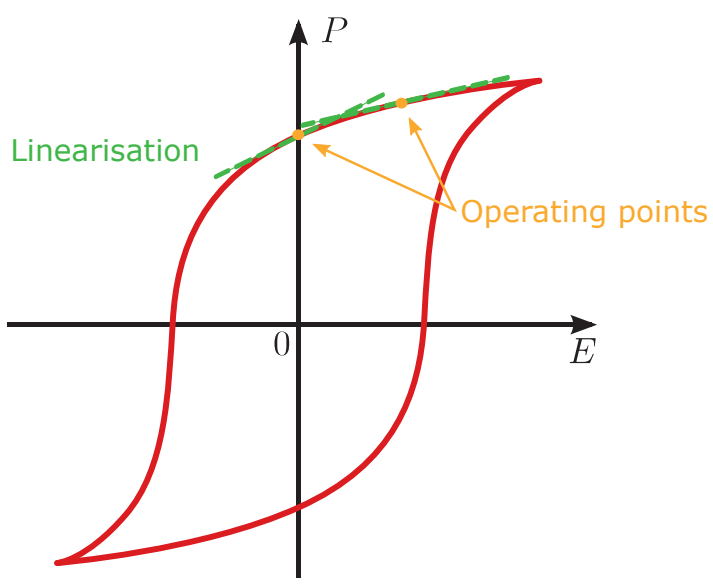

Figure 1: Exemplary hysteresis curve shows the relation between the polarisation $P$ and the electric field strength $E$ with a linearisation for the smallsignal behaviour in two operating points.

where $\boldsymbol{d}, \boldsymbol{s}^{\boldsymbol{E}}$ and $\varepsilon^{\boldsymbol{T}}$ are the coupling, the elastic compliance and the permittivity parameter matrices, respectively [7]. Taking into account the transversal isotropy and the resulting symmetry conditions, a total of ten independent material parameters are necessary, to describe the piezoelectric behaviour. In summary, three piezoelectric $\left(d_{15}, d_{31}, d_{33}\right)$, five elastic $\left(s_{11}^{\boldsymbol{E}}, s_{12}^{\boldsymbol{E}}, s_{13}^{\boldsymbol{E}}, s_{33}^{\boldsymbol{E}}, s_{44}^{\boldsymbol{E}}\right)$ and two dielectric $\left(\varepsilon_{11}^{\boldsymbol{T}}, \varepsilon_{33}^{\boldsymbol{T}}\right)$ material parameters are required. In order to determine all ten parameters, according to the IEEE Standard [7], several specimens of different shapes are required. The use of several specimens, however, entails uncertainties because the material properties are not consistent due to different production and polarisation processes. Since the specimens are piezoelectric discs poled in the thickness direction and with a much larger radius than thickness, the piezoceramic can be reduced to a 1D-model which is only sensitive to $s_{33}^{E}, \varepsilon_{33}^{T}$ and $d_{33}$. Mathematically this leads to a reduced set of equations:

$$
\begin{aligned}
& S_{3}=s_{33}^{E} T_{3}+d_{33} E_{3}, \\
& D_{3}=d_{33} T_{3}+\varepsilon_{33}^{T} E_{3} .
\end{aligned}
$$

If a piezoelectric ceramic is exposed to an alternating electric field, the electrical impedance $Z$ or admittance $Y$ is dependent on the frequency. The following procedure to determine the desired material parameters $s_{33}^{E}, \varepsilon_{33}^{T}$ and $d_{33}$ is based on the investigation of impedance measurements assuming a free resonator in thickness mode and of the fundamental analysation and the harmonics [7]. According to IEEE [7], at alternating electric fields, the admittance can be described as:

$$
Y(\omega)=\frac{1}{Z(\omega)}=\left(\frac{\mathrm{j} \omega \varepsilon_{33}^{S} \varepsilon_{0} A}{h}\right) \frac{1}{1-k^{2} \frac{\tan \left(\omega / 4 f_{\mathrm{p}}\right)}{\omega / 4 f_{\mathrm{p}}}}
$$

Here, $\omega$ is the angular frequency, $\varepsilon_{0}$ the dielectric constant of vacuum, $A$ the electrode area and $h$ the thickness of the specimen. $f_{\mathrm{p}}$ is the parallel resonance frequency of the first thickness mode, also known as the frequency of the maximum resistance and $\varepsilon^{S}$ is the permittivity at constant strain (clamped permittivity). The electromechanical coupling factor $k$ can be determined by

$$
k=\sqrt{\frac{\pi}{2} \frac{f_{\mathrm{s}}}{f_{\mathrm{p}}} \cot \left(\frac{\pi}{2} \frac{f_{\mathrm{s}}}{f_{\mathrm{p}}}\right)},
$$

with $f_{\mathrm{s}}$ as the series resonance frequency of the first thickness mode or frequency of the maximum conductance. By measuring the impedance of a piezoceramic it is possible to locate the characteristic frequencies $f_{\mathrm{s}}$ and $f_{\mathrm{p}}$ as well as to determine the free capacity $C_{0}$. In addition, the density $\rho$ is required which can be determined by measuring the mass and volume of the sample. Using these the linear parameters, $s_{33}^{E}, \varepsilon_{33}^{T}$ and $d_{33}$ can be estimated for different operating points.

\subsection{Non-linear approximation for mater- ial properties}

A DC bias approach to a characterisation of nonlinear material parameters of piezoelectric discs demands an extension of the constitutive equations by second order material parameters $[4,10]$. Once again for a one-dimensional approximation, equations 2 can be rewritten as

$$
\begin{aligned}
S_{3} & =s_{33}^{E} T_{3}+s_{333}^{E} T_{3}^{2}+d_{33} E_{3}+d_{333} E_{3} T_{3}+\zeta_{33} E_{3}^{2}, \\
D_{3} & =d_{33} T_{3}+d_{333} T_{3}^{2}+\varepsilon_{33}^{T} E_{3}+\zeta_{33} E_{3} T_{3}+\varepsilon_{333}^{T} E_{3}^{2},
\end{aligned}
$$

where $\varepsilon_{333}^{\boldsymbol{T}}, d_{333}$ and $\zeta_{33}$ are the non-linear dielectric, piezoelectric and electrostrictive material parameters, respectively. In Voigt notation, the subscript indices describe the direction of the inducing and resulting state variables. Since several inducing forces act on one non-linear material parameter, an additional index is necessary to describe the relation of the second order state variables (e.g. $E_{3}$ and $T_{3}$ act on $d_{333}$ resulting in $S_{3}$ ). Due to the superposition of a DC bias field $\bar{E}$ with a small AC field $\widetilde{E}$, the mechanical and electrical quantities are

$$
\begin{array}{ll}
E=\widetilde{E}+\bar{E}, & S=\widetilde{S}+\bar{S}, \\
D=\widetilde{D}+\bar{D}, & T=\widetilde{T} .
\end{array}
$$

It is noticeable that, because of the assumption of a mechanically free resonator, the stress $T$ has no contribution from a static component $\bar{T}$. According to $[3,4]$, the extended quantities in equation 6 can be inserted into equation 5 and split into two dynamic equations 


$$
\begin{aligned}
& \widetilde{S}_{3}=\left(s_{33}^{E}+d_{333} \bar{E}_{3}\right) \widetilde{T}_{3}+\left(d_{33}+\zeta_{33} \bar{E}_{3}\right) \widetilde{E}_{3}, \\
& \widetilde{D}_{3}=\left(d_{33}+\zeta_{33} \bar{E}_{3}\right) \widetilde{T}_{3}+\left(\varepsilon_{33}^{\boldsymbol{T}}+\varepsilon_{33}^{\boldsymbol{T}} \bar{E}_{3}\right) \widetilde{E}_{3},
\end{aligned}
$$

and two static equations

$$
\begin{aligned}
& \bar{S}_{3}=d_{33} \bar{E}_{3}, \\
& \bar{D}_{3}=\varepsilon_{33}^{T} \bar{E}_{3},
\end{aligned}
$$

for a DC field biased resonator. These static equations describe the mechanical extension and the dielectric displacement of the material as a result of an applied electrical DC bias field. However, it is important that the dynamic equations are now DC bias dependent and the relationship between the linear and non-linear parameters can be described as follows:

$$
\begin{aligned}
& s_{33}^{\mathrm{eff}}=\left(s_{33}^{E}+d_{333} \bar{E}_{3}\right)=s_{33}^{E}\left(1+\frac{d_{333}}{s_{33}^{E}} \bar{E}_{3}\right), \\
& d_{33}^{\mathrm{eff}}=\left(d_{33}+\zeta_{33} \bar{E}_{3}\right)=d_{33}\left(1+\frac{\zeta_{33}}{d_{33}} \bar{E}_{3}\right), \\
& \varepsilon_{33}^{\mathrm{eff}}=\left(\varepsilon_{33}^{T}+\varepsilon_{333}^{T} \bar{E}_{3}\right)=\varepsilon_{33}^{T}\left(1+\frac{\varepsilon_{333}^{T}}{\varepsilon_{33}^{T}} \bar{E}_{3}\right) .
\end{aligned}
$$

These material parameters, $s_{33}^{\text {eff }}, d_{33}^{\text {eff }}$ and $\varepsilon_{33}^{\text {eff }}$, have been determined by the linear approximation at different operating points. Therefore, the equations can be solved for the non-linear parameters.

\subsection{The DC bias field dependence of the resonance frequency}

Furthermore, the dependence of the resonance frequency on a DC bias field can be derived [3, 4]. A longitudinal acoustic wave velocity influenced by an applied DC bias field can be described as follows:

$$
v_{\mathrm{t}}\left(\bar{E}_{3}\right)=\sqrt{\frac{1}{s_{33}^{\text {eff }} \rho}} .
$$

Not only the elastic compliance $s_{33}^{\text {eff }}$ but also the density $\rho$ depend on the applied voltage. With the volume $V$ of the piezoelectric disc expanding in the same direction as the DC voltage assuming the 1D approximation, the mass $m$ of the disc remains constant. Thus $\rho$ can be written as

$$
\rho\left(\bar{E}_{3}\right)=\frac{m}{V\left(\bar{E}_{3}\right)}=\frac{m}{V_{0}\left[1+d_{33} \bar{E}_{3}\right]},
$$

with $V_{0}$ the volume without an applied DC bias field. According to [7] the wave velocity propagating in thickness direction of a disc shaped piezoceramic depends on the serial resonance frequency $f_{\mathrm{s}}$. Solving the equation for $f_{\mathrm{s}}$ and using the DC bias field dependent equations 13,12 and 11 yields

$$
f_{\mathrm{s}}\left(\bar{E}_{3}\right)=\frac{v_{\mathrm{t}}\left(\bar{E}_{3}\right)}{2 h}=f_{\mathrm{s}, 0}\left[1+\left(\frac{d_{33}}{2}-\frac{d_{333}}{2 s_{33}^{\mathrm{eff}}} \bar{E}_{3}\right)\right]
$$

where the change of the thickness $h=h_{0}\left(1+d_{33} \bar{E}_{3}\right)$ has been included. The index 0 indicates values without an applied DC bias field. By equation 14 a direct dependence of the resonance frequency on the applied electrical field is given. Accordingly, a change of the frequency with and without applied DC voltage can be described as follows:

$$
\frac{\Delta f_{\mathrm{s}}}{f_{\mathrm{s}, 0}}=\frac{d_{33}}{2}-\frac{d_{333}}{2 s_{33}^{\mathrm{eff}}} \bar{E}_{3} .
$$

The first term describes the shift of the series frequency due to the mechanical deformation of the piezoceramic disc. The second term describes the part of the resonance shift caused by the non-linear behaviour.

\section{Measurement system}

The measurement setup depicted in figure 2 is used to determine the frequency dependent impedance of various piezoelectric specimens under high electric fields. A DC bias test fixture connects the sample to a DC voltage source (EA-PSI 9200-04T power supply) and the impedance analyser (Keysight E4990A). The design of the fixture is based on a protection circuit for the impedance analyser recommended by the manufacturer [11]. It supports an external voltage as well as a frequency range of $35 \mathrm{kHz}$ to $20 \mathrm{MHz}$. The DC source provides voltages up to $200 \mathrm{~V}$ which is sufficient to apply an electric field strength up to $1 \frac{\mathrm{kV}}{\mathrm{mm}}$, depending on the thickness of the samples.

All examined piezoceramics are disc-shaped with full-surface silver electrodes manufactured by Physik Instrumente (PI) Ceramic $\mathrm{GmbH}$ [12]. In total, three different types of piezoceramics were investigated. PIC255 is a piezoceramic based on soft PZT with a radius of $r=8 \mathrm{~mm}$ and thickness of $h=0.2 \mathrm{~mm}$. PIC181 is a hard PZT piezoelectric ceramic with equal dimensions. PIC700 is a lead-free piezoceramic material based on bismuth sodium titanate with coupling factors and permittivities comparable to piezoceramics based on barium titanate, according to the manufacturer [12]. During the investigations, PIC700 was not available with the same dimensions as the other specimen, therefore the lead-free ceramic has a thickness of $h=0.5 \mathrm{~mm}$ and a radius of $r=5 \mathrm{~mm}$

\section{Results}

\subsection{Measurement results}

As described in section 2, impedance spectra of the specimen measured near the resonance frequencies $f_{\mathrm{s}}$ and $f_{\mathrm{p}}$ can be used to determine the linear material parameters $s_{33}^{E}, \varepsilon_{33}^{T}$ and $d_{33}$. All samples are mounted as shown in figure 2 and the applied electric field strength is gradually changed from $1 \frac{\mathrm{kV}}{\mathrm{mm}}$ to $-1 \frac{\mathrm{kV}}{\mathrm{mm}}$ 
DC voltage source

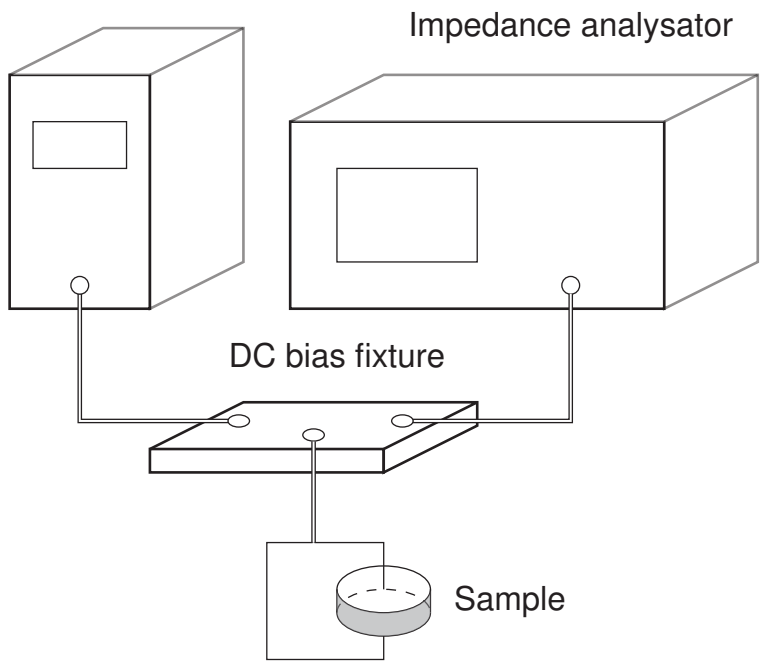

Figure 2: Schematic of the measurement setup.

and back again. Figure 3 depicts the measured relation between the magnitude of the impedance $|Z|$ and the resonance frequency $f$ of the PIC255 at different operating points. The black markers indicate the detected series resonance frequency at different bias field strengths and visually underline the shift of $f_{\mathrm{s}}$. Moreover a general trend of the shift to higher frequencies with an electric field applied along the polarisation direction is recognizable. And vice versa the frequencies $f_{\mathrm{s}}$ and $f_{\mathrm{p}}$ shift to lower frequencies with negative (against the polarisation direction) electric field strengths applied. The maximum $\Delta f_{\mathrm{s}}$ of PIC255, measured with an applied electric field strength of $1 \frac{\mathrm{kV}}{\mathrm{mm}}$, is $\approx 37 \mathrm{kHz}$. It should be mentioned that for correct mathematical estimation of the linear material parameters, $f_{\mathrm{s}}$ is detected as the maximum weighted conductance $\max (\operatorname{Re}\{Y(\omega) / \omega\})$ and $f_{\mathrm{p}}$ as the maximum weighted resistance $\max (\operatorname{Re}\{\omega Z(\omega)\})[3,13]$. Figure 4 depicts the relation between $|Z|$ and $f$ for PIC255 with applied negative electric field strengths. Using $E$ greater than $-800 \frac{\mathrm{V}}{\mathrm{mm}}$, the piezoceramic material depolarises. This state remains, even if the negative field is switched off. Yang et al. observed a similar material depoling of soft PZT piezoelectric samples EC-65 and EC-76 at field strengths of $-800 \frac{\mathrm{V}}{\mathrm{mm}}$ and $-500 \frac{\mathrm{V}}{\mathrm{mm}}$, respectively [14]. However, the piezoelectric ceramic can be re-polarised by applying a positive electric field strength of $1 \frac{\mathrm{kV}}{\mathrm{mm}}$. This is evident when considering the material parameters as a function of the applied DC bias field in subsection 4.3.

In general, PIC181 shows the same frequency shift behaviour as PIC255 when an electric field is applied, although the effect is smaller $\left(\Delta f_{\mathrm{s}} \approx 27 \mathrm{kHz}\right)$. Moreover this piezoceramic disc doesn't depolarise at negative bias field strengths up to $-1 \frac{\mathrm{kV}}{\mathrm{mm}}$. The lead-free piezoceramic PIC700 also appears to be less susceptible to the applied field. However, it should be noted that

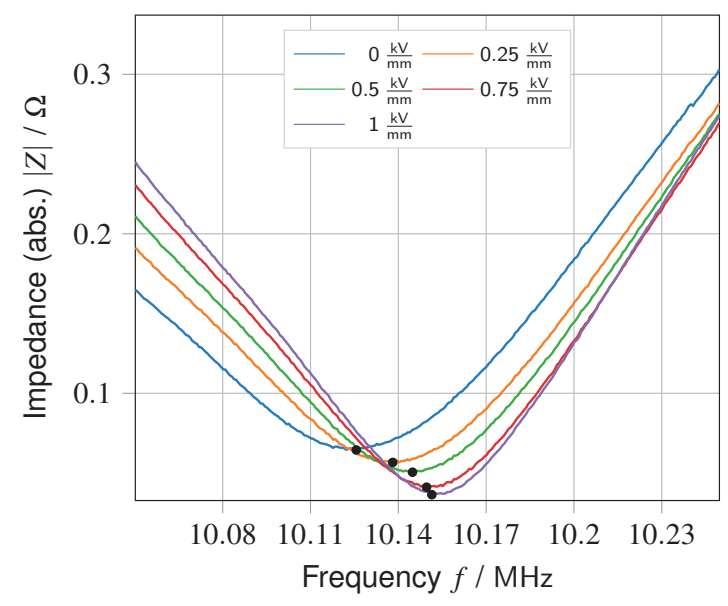

Figure 3: The relation between the absolute value of the impedance $|Z|$ and frequency $f$ of PIC255 at various positive $D C$ bias fields applied. Series resonance frequency is marked by black dots.

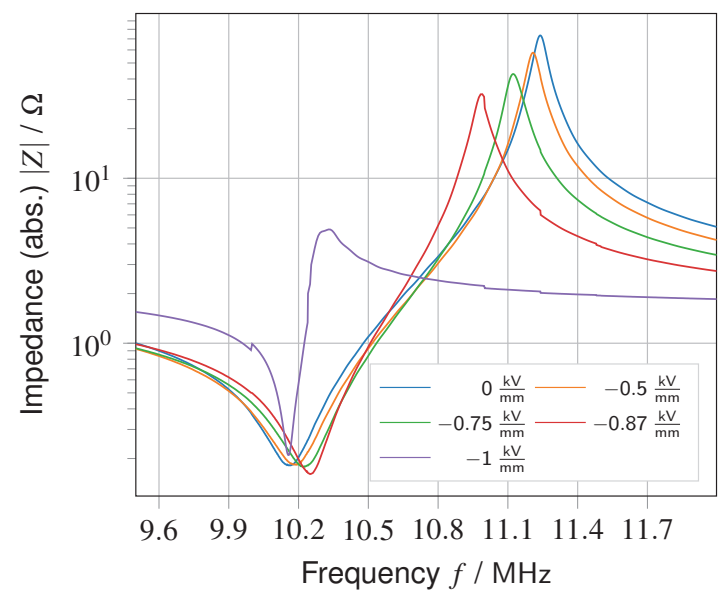

Figure 4: The relation between the absolute value of the impedance $|Z|$ and frequency $f$ of PIC255 at various negative $D C$ bias fields applied. Depolarisation occurring at $-1 \frac{\mathrm{kV}}{\mathrm{mm}}$.

due to the geometry $(h=0.5 \mathrm{~mm})$ only maximum DC bias field strengths of $\pm 400 \frac{\mathrm{V}}{\mathrm{mm}}$ are applicable. In the aforementioned field range, a maximum $\Delta f_{\mathrm{s}}$ of $\approx 1 \mathrm{kHz}$ can be measured for all examined PIC700 ceramics and is comparable to the magnitude of the shift of PIC181 at $\pm 400 \frac{\mathrm{V}}{\mathrm{mm}}$, but thinner samples are necessary for a reliable statement. The general shift shows an opposite trend in the aforementioned field strength range, thus $f_{\mathrm{s}}$ and $f_{\mathrm{p}}$ shift to lower frequencies at greater DC bias fields and vice versa.

\subsection{Frequency shift by deformation}

At this point it is important to verify that the frequency shift is caused by the non-linear behaviour and not the material deformation. For this purpose, equation 15 can be used to determine a $\Delta f_{\mathrm{s} \text {, mech }}$ which is solely 
dependent on mechanical deformation.

$$
\Delta f_{\mathrm{s}, \text { mech }}=f_{\mathrm{s}, 0} \frac{d_{33}}{2} \bar{E}_{3}
$$

The calculated value should be lower than the measured value $\Delta f_{\mathrm{s}}\left(\bar{E}_{3}\right)$. For PIC255 the $\Delta f_{\mathrm{s}, \mathrm{mech}}=12.1 \mathrm{kHz}$ and for PIC181 $\Delta f_{\text {s,mech }}=8.7 \mathrm{kHz}$. Both values are below the aforementioned frequency shift, so it can be claimed that the present effect is caused primarily by the DC bias field and non-linear behaviour of the piezoceramics.

\subsection{Linear parameter estimation}

So far, only the influence of the applied DC bias field on the absolute value of the impedance has been analysed and compared. The change of the elastic compliance as a function of the applied DC bias field of PIC255 and PIC181 is depicted in figure 5. Both piezoelectric samples were exposed to the same electrical stress cycle. As expected, the piezoceramic PIC181 seems to be less influenced by a bias field, because the changes in the elastic compliance are small compared to PIC255. Nevertheless the shift of material parameter of both piezoceramics shows a trend in the same direction. As the DC bias field decreases, the elastic compliance of both piezoceramics increases.

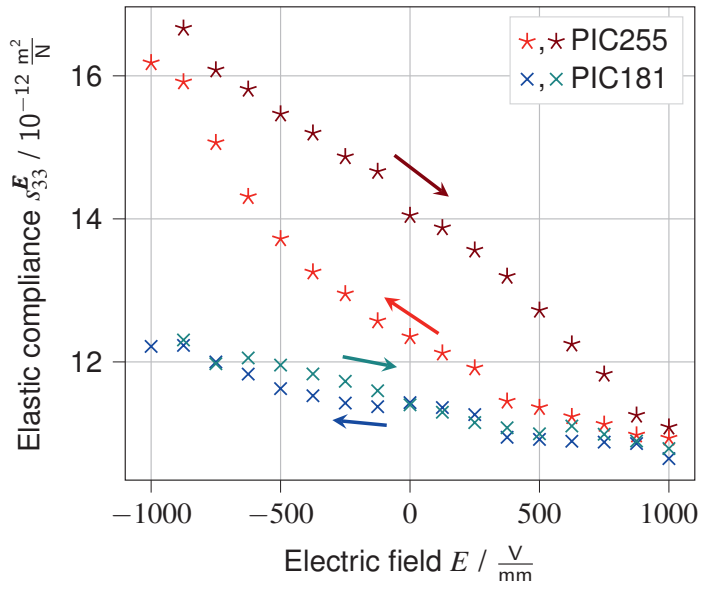

Figure 5: Elastic compliance $s_{33}^{E}$ of PIC255 and PIC181 at different operating points.

A similar behaviour for $d_{33}$ and $\varepsilon_{33}^{T}$ is shown in figure 6 and 7 , respectively. While the piezoelectric parameter and the permittivity of PIC255 change significantly, they remain relatively constant in PIC181. It is not surprising that the trend of both aforementioned material parameters is proportional because the permittivity is necessary to estimate the piezoelectric constant [7]. Another effect which occurs with all material parameters of the PIC255 is a discontinuity of the parameters at high negative field strengths. Due to depolarisation of PIC255, the characteristic resonance frequencies are only poorly indicated and a reliable determination of $d_{33}, s_{33}^{E}$ and $\varepsilon_{33}^{T}$, which is based on the detection of $f_{\mathrm{s}}$ and $f_{\mathrm{p}}$, is impossible. Moreover the observed significant decrease of $d_{33}$ and $\varepsilon_{33}^{T}$ at $E=-1 \frac{\mathrm{kV}}{\mathrm{mm}}$ remain low until a positive field strength of more than $500 \frac{\mathrm{kV}}{\mathrm{mm}}$ is applied to the soft PZT sample.

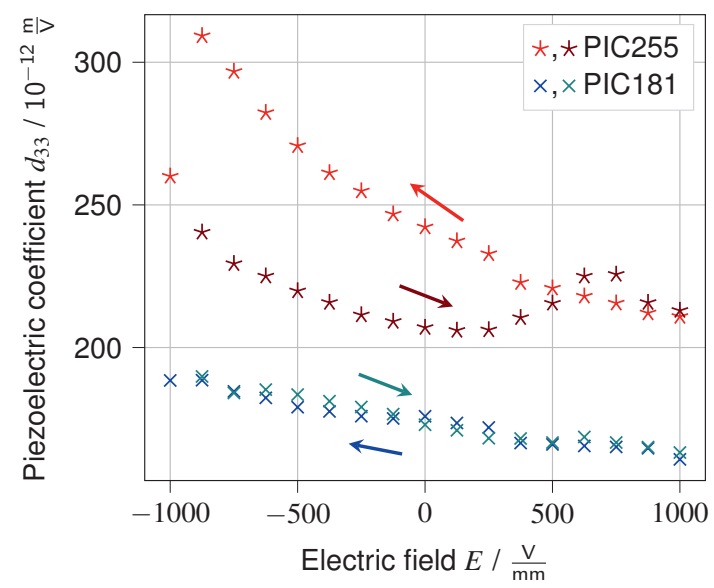

Figure 6: Piezoelectric coefficient $d_{33}$ of PIC255 and PIC181 at different operating points.

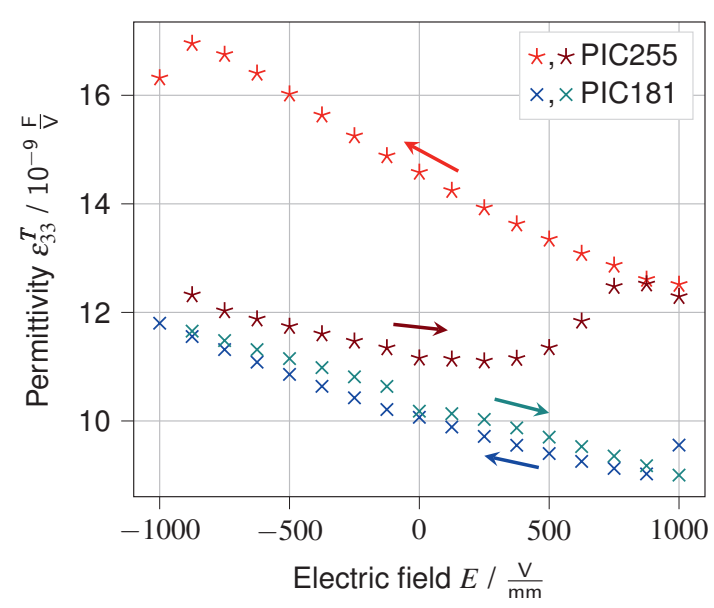

Figure 7: Permittivity $\varepsilon_{33}^{T}$ of PIC255 and PIC181 at different operating points.

Although the frequency shift direction of PIC700 is opposite to the other PZT based piezoceramics, the material parameters show the same characteristics in the considered field strength range. The relation between the compliance $s_{33}^{E}$ and the applied DC bias field of three different PIC700 samples is shown in figure 8 . The parameter change is comparably small to that of PIC181. Moreover if the DC bias field decreases, $s_{33}^{E}$ increases. This behaviour is also observed for the piezoelectric material parameter and the permittivity.

The application of a DC bias field in the direction of polarisation reinforces the remenant polarisation, which leads to a decrease in elastic compliance and the piezoelectric coefficient. According to [3] this can be explained considering the $\boldsymbol{P}-\boldsymbol{E}$ hysteresis (figure 1). When a DC bias field is superimposed with an 


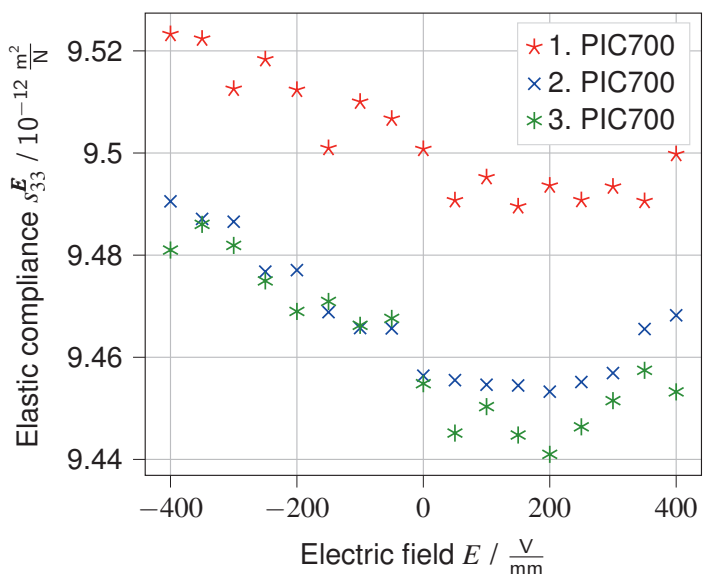

Figure 8: Elastic compliance $s_{33}^{E}$ of three PIC700 samples at different operating points.

AC alternating field and applied to a piezoceramic disc, $\varepsilon_{33}^{\text {eff }}$ describes the slope of the $\boldsymbol{P}$ - $\boldsymbol{E}$ field curve $(d \boldsymbol{P} / d \boldsymbol{E})$ at a variable operating point. As the DC bias field strength increases, the slope decreases, causing $\varepsilon_{33}^{\text {eff }}$ to decrease as well. Since the piezoelectric parameter $\boldsymbol{d}$ is approximately proportional to $\boldsymbol{\varepsilon}^{T}$, the DC bias field causes $\boldsymbol{\varepsilon}^{T}$ to decrease.

\subsection{Non-linear parameter estimation}

By using equations 5 the non-linear parameters can be estimated. Considering the maximum applicable electrical field strength of $1 \frac{\mathrm{kV}}{\mathrm{mm}}$ the following values result for the piezoelectric, electrostrictive and dielectric parameter of PIC255:

$$
\begin{aligned}
& d_{333}=-7.2 \cdot 10^{-20} \mathrm{~m}^{3} / \mathrm{VN} \\
& \zeta_{33}=-17.7 \cdot 10^{-19} \mathrm{~m}^{2} / \mathrm{V}^{2} \\
& \varepsilon_{333}^{T}=-206.2 \cdot 10^{-17} \mathrm{~F} / \mathrm{V}
\end{aligned}
$$

The following parameters can be estimated for PIC181:

$$
\begin{aligned}
& d_{333}=-6.1 \cdot 10^{-20} \mathrm{~m}^{3} / \mathrm{VN} \\
& \zeta_{33}=-19.6 \cdot 10^{-19} \mathrm{~m}^{2} / \mathrm{V}^{2} \\
& \varepsilon_{333}^{T}=-51 \cdot 10^{-17} \mathrm{~F} / \mathrm{V}
\end{aligned}
$$

Because of the different thickness of the lead-free piezoceramic specimen a direct comparison is not possible. Moreover the observed parameter changes in the DC bias range from $-400 \frac{\mathrm{v}}{\mathrm{mm}}$ to $400 \frac{\mathrm{V}}{\mathrm{mm}}$ are too small for a reliable assumption. The measured frequency shift of all piezoceramic discs is comparable with similar experiments $[3,8,9,14]$.

\section{Conclusions}

Impedance measurements of three different piezoceramic materials with various DC bias field strengths applied were performed. A series and parallel resonance frequency shift is recognisably caused by the applied field strength. A frequency shift trend to higher frequencies with an electric field applied along the polarisation direction is detected. The general trend of the frequency shift of the lead-free piezoceramic disc is opposite to the shift of the leaded specimens. Nevertheless the parameter change of PIC700 is comparable to that of the hard PZT piezoceramic PIC181. The investigations of the material parameters shows an almost linear dependence of the parameters and the applied DC bias field, comparable with other publications in this field $[3,4,8$, $9,14]$. The linear elastic parameter $s_{33}^{E}$, the permittivity $\varepsilon_{33}^{T}$ and the piezoelectric constant $d_{33}$ decrease proportionally with increasing electric field strength $E$. Furthermore, depolarization effects are observed at the soft PZT PIC255 by applying a DC bias field of greater than $-800 \frac{\mathrm{V}}{\mathrm{mm}}$. A 1D-approximation is described to determine non-linear parameters of piezoelectric discs. Second-order constitutive equations provide results for the non-linear piezoelectric coefficient $d_{333}$, the permittivity $\varepsilon_{333}^{T}$ and the electrostrictive coefficient $\zeta_{33}$, comparable to [3].

\section{$6 \quad$ Literature}

[1] M. Alguero, C. Alemany, L. Pardo, A. M. Gonzalez, "Method for Obtaining the Full Set of Linear Electric, Mechanical, and Electromechanical Coefficients and All Related Losses of a Piezoelectric Ceramic", Journal of the American Ceramic Society 87.2, pp. 209-215 (2004); doi: 10.1111/j.15512916.2004.00209.x

[2] Y. Dong, Z. Wu, H. Hu, B. Wu, G. Xu, "A novel method for characterization of piezoelectric material parameters by simulated annealing optimization", IEEE Transactions on Ultrasonics, Ferroelectrics, and Frequency Control 57.12, pp. 2613-2615 (2010); doi: 10.1109/TUFFC.2010.1735

[3] Q.-M. Wang, T. Zhang, Q. Chen, X.-H. Du, "Effect of DC bias field on the complex materials coefficients of piezoelectric resonators", Sensors and Actuators A: Physical 109.1, pp. 149-155 (2003); doi: 10.1016/j.sna.2003.08.008

[4] K. S. Aleksandrov , M. P. Zaitseva , A. M. Sysoev, Yu. I. Kokorin, "The piezoelectric resonator in a dc electric field", Ferroelectrics 41.1, pp. 3-8 (1982); doi: 10.1080/00150198208210604

[5] W. P. Mason, "Electromechanical transducers and wave filters", New York: D. Van Nostrand Co., 1948.

[6] D. S. Shim, D. A. Feld, "A general nonlinear Mason model of arbitrary nonlinearities in a piezoelectric film", IEEE International Ultrasonics Symposium, pp. 295300(2010); doi: 10.1109/ULTSYM.2010.5935834

[7] American National Standards Institute, "IEEE Standard on Piezoelectricity", New York, 1987; doi: 10.1109/IEEESTD.1988.79638

[8] R. Simkovics, H. Landes, M. Kaltenbacher, R. Lerch, "Nonlinear finite element analysis of piezoelectric transducers", IEEE Ultrasonics Symposium 
2, pp. $1057-1060 \quad$ (1999); doi: 10.1109/ULTSYM.1999.849182

[9] F. Li, Z. Xu, X. Wei, X. Yao, "Temperature- and DC Bias Field-Dependent Piezoelectric Effect of Soft and Hard Lead Zirconate Titanate Ceramics", Journal of Electroceramics 24, pp. 294-299; doi: 10.1007/s10832-0099571-1

[10] R. Simkovics, "Nichtlineares piezoelektrisches FiniteElemente-Verfahren zur Modellierung piezokeramischer Aktoren", Düsseldorf: VDI-Verl, 2002.

[11] "Impedance Measurement Handbook", Santa Rosa, USA: Keysight Technologies, Nov. 2016

[12] "Piezoelectric Ceramic Products: fundamentals, characteristics and applications", Physik Instrumente (PI) GmbH \& Co.KG, Dez. 2016

[13] S. Sherrit, H D. Wiederick und B.K. Mukherjee, "Non-Iterative Evaluation of the Real and Imaginary Material Constants of Piezoelectric Resonators", Ferroelectrics 134, pp. 111-119 (1992); doi: 10.1080/00150199208015574

[14] G. Yang, W. Ren, S.-F. Liu, A. J. Masys, B. K. Mukherjee, "Effects of uniaxial stress and DC bias field on the piezoelectric, dielectric, and elastic properties of piezoelectric ceramics", IEEE Ultrasonics Symposium 2, pp. 1005-1008 (2000); doi: 10.1109/ULTSYM.2000.921494 